\title{
Movimiento social, nuevas formas de hacer política y enclaves autoritarios. Los debates del Consejo Asesor para la Educación en el gobierno de Michelle Bachelet en Chile ${ }^{1}$
}

\author{
Manuel Antonio Garretón \\ Universidad de Chile, Santiago, Chile. \\ Email: mgarreton@mi.cl \\ María Angélica Cruz \\ Universidad de Valparaíso, Valparaíso, Chile. \\ Email: angecruz@hotmail.com
}

Félix Aguirre

Universidad de Valparaíso, Valparaíso, Chile.

Email: felix.aguirrediaz@gmail.com

Naim Bro

Universidad de Chile, Santiago, Chile.

Email: naim.bro@ug.uchile.cl

Elías Farías

Universidad de Chile, Santiago, Chile.

Email: elias.fariasc@gmail.com

\section{Pierina Ferreti}

Universidad de Valparaíso, Valparaíso, Chile.

Email: pierinaferretti@gmail.com

\section{Tamara Ramos}

Universidad de Lovaina, Lovaina, Bélgica.

Email: tamaramosmerino@gmail.com

Resumen: A partir de una investigación sobre los Consejos Asesores Presidenciales en el Gobierno de Michelle Bachelet, se desarrolla un marco teórico sobre movimientos sociales y las llamadas formas subpolíticas, que permite ver cómo el movimiento estudiantil secundario de 2006 desafió el enclave educacio- 
nal y el tipo de respuesta que un gobierno que buscaba un sello ciudadano, dio a través de la formación de un Consejo Asesor con participación de diversos sectores. El análisis de los debates en el seno de ese Consejo muestra las diversas posiciones en juego y también la imposibilidad de llegar a acuerdos de trasformación profunda.

Palabras clave: Modelo educacional, movimiento estudiantil, Consejos Asesores, subpolítica, políticas públicas, enclaves autoritarios

\title{
Social movement, new ways of political action and authoritarian enclaves. The debates of the Advisory Council for Education in the government of Michelle Bachelet in Chile
}

\begin{abstract}
Based on an investigation on the Presidential Advisory Council on the government of Michelle Bachelet, is developed here a theoretical framework on social movements and the so called subpolitical ways, that allows to see how the student's movement of 2006 challenged the secondary level educational enclave, and the kind of response a government that was seeking for a citizen's seal gave through the formation of an Advisory Council with the participation of various sectors. The analysis of the debates within the Council show the various positions al stake and also the inability to reach agreements towards a profound transformation.
\end{abstract}

Keywords: educational model, student movement, Advisory Councils, subpolitics, public policy, authoritarian enclaves

\section{Movimento social, novas formas de fazer política e enclaves autoritários. As discussões do Conselho Consultivo de Educação no governo de Michelle Bachelet no Chile}

Resumo: Baseado em uma investigação sobre o Conselho Presidencial Consultivo em o governo de Michelle Bachelet, desenvolve um quadro teórico sobre movimentos sociais e as chamadas formas subpoliticas que permitem ver como o movimento estudantil de 2006 desafiou o enclave de ensino secundário e como o tipo de resposta do governo em procura de um selo cidadão, foi através da formação de um Conselho Consultivo com a participação de vários setores. A análise dos debates no seio do Conselho mostra as várias posições no jogo e também a incapacidade de chegar a acordos de profunda transformação.

Palavras-chave: modelos de ensino, movimento estudantil, Conselhos Consultivos, subpolítica, Política pública, enclaves autoritários

$$
* * *
$$

\section{Presentación}

Entre los debates principales de la sociedad chilena post transición (1990 en adelante), heredera de muchos elementos de la dictadura militar, ha estado la cuestión educacional. La movilización de estudiantes secundarios, en 2006, conocida como "revolución de los pingüinos"2 , junto a la 
solución parcial encontrada a ese conflicto con la creación del Consejo Asesor Presidencial para la Calidad de la Educación (CAPCE), constituyó un hito fundamental en dicho debate. En el marco de una investigación más amplia sobre movilizaciones sociales, Consejos Asesores Presidenciales y conocimiento experto, nos preguntamos en este artículo por el tipo de movimiento social que constituían las movilizaciones estudiantiles y por la naturaleza de la solución encontrada. A nivel más conceptual nos preguntamos por el papel de los movimientos sociales en la formulación de políticas públicas en un contexto post transicional y por si esta forma de resolución de conflictos y de gobernar implican una transformación de la política clásica en la conformación de una ciudadanía más activa en la toma de decisiones.

\section{Política y subpolítica}

Hay tres cuestiones conceptuales de tipo general y una de contexto referida a la sociedad chilena que están en juego. La primera cuestión en debate tiene que ver con las transformaciones de la política en la época contemporánea.

En este sentido, Beck (1998) señala que la modernidad reflexiva apela a una suerte de autoconfrontación respecto a las consecuencias que acarrea la sociedad del riesgo, las cuales son internalizadas por los individuos provocando la desestabilización de los fundamentos del orden social. Toda decisión política, al implicar riesgos para la sociedad, puede tornarse en un motivo de deslegitimación y rechazo al sistema de normas establecido y, sobre todo, a la legitimidad del control por parte de las instituciones.

En este contexto surgen iniciativas ciudadanas que se toman el poder temáticamente, conformando la agenda política en forma contingente. Los petitorios personales de individuos, conviven con los petitorios de los actores sociales y colectivos. "[...] Nos encontramos con cada vez más problemas en situaciones que no pueden ser comprendidas ni adecuadamente resueltas con las actuales instituciones e ideas, con las concepciones vigentes de lo político" (Ibid:16-17) donde "[...] la falta de soluciones del ayer, junto con la falta de soluciones del anteayer, claman por el dominio de un futuro totalmente fuera de los marcos conocidos” (Ibid:18).

El concepto de "subpolítica” expresa tal fenómeno, distinguiéndose de las formas tradicionales de hacer política en la medida en que se canaliza por vías alternativas a las institucionales. Los orígenes de este concepto remiten a Beck, quien plantea que en el contexto del paso entre una sociedad moderna a una sociedad reflexiva, "La subpolítica se diferencia de la política por el hecho de que: (a) los actores aparecen en el escenario de la formación social, fuera del sistema político o corporativo (...), y (b) por el hecho de que no sólo los actores sociales y colectivos, sino también individuos compiten con ellos y entre sí por el emergente poder de estructuración de lo político” (Ibid: 140-141). 
Por su parte, Holzer y Sørensen (2001) agregan complejidad al concepto, afirmando que si bien la subpolítica suele entenderse como aquella política “debajo" del Estado Nación, también puede ser conceptualizada como una forma de política "fuera y más allá" de las instituciones representativas del sistema político del Estado Nación (Ibid: 18), de forma que el prefijo $s u b$ no refiere a la relevancia, sino que al menor grado de institucionalización en relación a la política tradicional y a una renuncia a los canales formales, es decir, a la política institucionalizada. Este concepto de subpolítica también se ha entendido como aquellas decisiones que serían no-políticas, que han sido tomadas en esferas como la economía y la ciencia, pero que conllevan consecuencias substanciales sobre la sociedad (Beck, 1998). Estamos en una sociedad donde todo aquello que se daba por sentado ahora es cuestionado, permitiendo que una serie de elementos o cosas que anteriormente no eran políticas, ahora sí lo sean. En la medida en que "se perciben ahora como contingentes, ellos pueden ser puestos en duda (o desafiados) y por lo tanto pueden ser objeto de luchas políticas" (Holzer y Sørensen, 2001: 4). Holzer y Sorensen, abogan por un concepto comprehensivo de subpolítica, y establecen una distinción entre la que se refiere a aquellos actos externos al sistema político tradicional que intencionalmente buscan ejercer poder y cambiar en alguna forma la condición de la sociedad, que se dan por sentado, a lo que llamarán subpolítica activa. Mientras que a la subpolítica referida a aquellas fuentes no-estatales de poder que moldean la sociedad sin buscarlo necesariamente de forma explícita, le llamarán subpolítica pasiva.

Estos dos conceptos son útiles para nuestro análisis, en la medida en que los autores asocian la subpolítica activa a los movimientos sociales y la subpolítica pasiva a los expertos que toman decisiones en ámbitos que tienen impacto sobre las personas. Pero también como veremos, y en esto modificamos en parte esta distinción, la sub-política activa obliga a respuestas desde los actores estatales que llevan a nuevas subpolíticas pasivas.

\section{Acción colectiva y movimientos sociales}

La segunda cuestión conceptual se refiere a la definición de los movimientos sociales. Hay aquí dos aspectos.

Por un lado, el concepto de política contenciosa alude al "levantamiento de demandas colectivas que interpela intereses de otras personas e implica al gobierno como demandante, objeto de demandas o terceras partes” (Tilly y Tarrow, 2007: 23, t. p.). Si para Beck, como hemos dicho, la subpolítica poseía grados menores de institucionalización en relación a la política tradicional, Tilly y Tarrow diferencian entre el tipo de "política contenciosa contenida" y el tipo de "política contenciosa transgresiva" (Mc Adam, Tarrow y Tilly, 2004). En el primer tipo -la contenida- los actores que levantan las demandas están previamente institucionalizados y cuentan con mecanismos de formulación de demandas también institucionalizados. 
En cambio, el segundo tipo de política contenciosa -la transgresiva-corresponde al caso cuando al menos uno de los actores no estaba previamente institucionalizado y tampoco sus formas de levantar demandas.

La "estructura de oportunidades" también es un concepto importante en la propuesta de estos autores. Se define según la interacción recurrente entre los actores políticos y el gobierno, viéndose condicionada por aspectos del régimen político, tales como la multiplicidad de centros de poder independientes, la apertura a nuevos actores, la inestabilidad de los alineamientos políticos, la existencia de aliados influyentes por parte de los desafiantes y el grado en que el régimen reprime o no los actos contenciosos (Tilly y Tarrow, 2007: 57). Por ende, debe entenderse que las formas de la acción contenciosa tienden a variar de acuerdo a los tipos de regímenes, los cuales se caracterizan por las relaciones regulares entre gobiernos, actores políticos establecidos, desafiantes, y actores políticos exógenos, pudiendo también ser otros gobiernos. En este sentido, la "capacidad del régimen” y el "grado de democracia” incidirán en la forma de operación de la acción contenciosa, en el entendido que la "capacidad" refiere al grado en el que las acciones del gobierno afectan la distribución de población, recursos, y actividades dentro del territorio del gobierno; mientras que el "grado de democracia”, se evalúa en función de la igualdad de los derechos políticos de la gente, la igual capacidad de influir en las políticas públicas y la protección de la acción arbitraria del Estado. Así, cada régimen establece límites a la forma en que la política contenciosa puede ser efectuada, además de tener una determinada capacidad para hacer cumplir estos límites, que se mueven entre lo prescrito, lo tolerado y prohibido (Tilly y Tarrow, 2007: 61).

Por otro lado, en referencia los movimientos sociales, podemos definirlos como un tipo de acción colectiva -no la única forma de acción colectiva- que posee una cierta densidad organizacional, una cierta duración en el tiempo y que se plantea la conservación o la transformación de la sociedad en su conjunto, o de un ámbito dentro de ella. Ahora, en lo que llamamos movimientos sociales hay tres dimensiones que interesa destacar por su pertinencia para el caso chileno. Por un lado, está aquel tipo de acción que tiene al movimiento de algún modo como su propio referente, es decir, el sentido de la acción es la constitución de un sujeto. Hay una segunda dimensión que es la dimensión instrumental o reivindicativa, que puede referirse a demandas o medidas concretas o que puede tener como objeto intentar fortalecer la organización. Y la tercera dimensión es la histórico-política, que algunos autores denominan historicidad, es decir, aquella orientación que va más allá de la demanda concreta, y que apunta a la transformación de la sociedad o de un ámbito de ella.

Así, puede introducirse una distinción entre lo que podríamos el Movimiento Social Central de una sociedad o época, que define el conflicto o problemática central de ellas apuntando a superarlos en un nueva sociedad (historicidad), y los movimientos sociales particulares que definen una problemática específica (instrumental o constitutiva de un deter- 
minado sujeto), en que la relación entre ambos es una hipótesis teórica básica, pero que depende cada sociedad y que requiere investigación empírica. ${ }^{3}$.

En el último tiempo el concepto de movimiento social ha sido subsumido en el de sociedad civil, concepto demasiado amplio que engloba tanto las dimensiones de actores y movimientos, ciudadanía y tejido asociativo $^{4}$. Para algunos autores clásicos la sociedad civil incluye el mercado, para otros sólo la parte "virtuosa" de la sociedad opuesta generalmente al Estado. Sin entrar en esta discusión ni en la taxonomía de lo que abarca el concepto, puede indicarse que la sociedad civil refiere a la larga o mediana duración de una sociedad, en cambio, los movimientos sociales corresponden más a un fenómeno temporal. Si la sociedad civil viene a ser algo así como el sustrato en que se constituyen los movimientos sociales, lo que importa entonces es el análisis sobre el modo cómo ella -los sujetos y actores sociales- se constituye en cada contexto histórico. Ello obliga a una hipótesis general de su constitución en cada caso histórico, pues las sociedades civiles no son lo mismo en todas partes, como veremos más adelante para el caso chileno.

\section{Políticas públicas y representación de intereses}

La tercera cuestión conceptual se refiere a las políticas públicas y al modo cómo ellas se vinculan a la representación de los intereses de los sectores afectados ${ }^{5}$. En el tratamiento de este tema ha predominado una visión tecnocrática que define un actor, el Estado, que responde racionalmente ya sea a su propia agenda o a demandas provenientes de diversos sectores sociales, a partir de la aplicación de lo que se llama el conocimiento experto. Se trata de romper esta visión analizando las políticas públicas como resultado de la interacción entre la sociedad, incluidos los eventuales movimientos sociales, y los actores estatales

Así, cada vez con mayor frecuencia se busca incluir a la ciudadanía en el proceso de la toma de decisiones en los asuntos de interés general, tanto sobre los temas a abordar como en el diseño y la aplicación de políticas públicas. No se buscaría con ello sólo mejorar la mecánica y la organización de las decisiones democráticas, sino que la participación del "público" garantiza la elaboración de una agenda pública que potencia la satisfacción de un conjunto de nuevas necesidades sociales y además es un antídoto ideal que reduce la creciente "incertidumbre" que caracteriza la vida colectiva (Beck, 1998; Castel, 2004). En este sentido, no sólo se ven afectados los actores sociales, sino también aquellos grupos que se podrían identificar como expertos, puesto que al decir de Giddens (1998) la sociedad contemporánea hace un buen tiempo que dejó de confiar su orden normativo a la acumulación del conocimiento científico, de forma que los expertos parecen no tener respuesta a esta crisis de racionalidad. Dicho de otro modo, los expertos no pueden seguir garantizando certidumbres con respecto a los diferentes riesgos tecnológicos y sociales, estando obligados 
a compartir sus dudas con la sociedad (Giddens, 1998). Puede pensarse que los Consejos Asesores Presidenciales, uno de los cuales es el objeto de estudio de este artículo, han sido una de estas instancias donde confluyen personas vinculadas a la academia y la ciencia (expertos), políticos, representantes de la sociedad civil, en la elaboración de políticas públicas, de modo de fortalecer una democracia en que los poderes públicos interactúan con la sociedad.

\section{Transformaciones de la sociedad chilena}

La cuestión de contexto se refiere a los cambios ocurridos en los últimos años en la sociedad chilena y aquí corresponde recordar como hipótesis analítica, reiterada en otros trabajos, el modo cómo en Chile se constituían los actores sociales y, si se quiere, la sociedad civil ${ }^{6}$. La "columna vertebral" de Chile correspondía a "un sistema de articulación de los sujetos y actores sociales en referencia al Estado [...] a partir de un tejido de relaciones entre organizaciones de la sociedad civil y estructura política partidaria” (Garretón, 1983: 32). No obstante, este sistema que hemos llamado matriz político-céntrico o estatal nacional popular a nivel latinoamericano, y en el caso chileno añadimos democrático y partidario se vio debilitado desde la década de los setenta por los regímenes militares y el modelo socio-económico neo liberal. Ello significó básicamente la pérdida del papel del Estado y los partidos políticos como espacios aglutinadores de sentidos de la comunidad política (Lechner, 1994), lo que también ha sido descrito como un desplazamiento de un imaginario público a un imaginario privado (PNUD, 2002). Según el PNUD, esta erosión de los imaginarios colectivos y el predominio de los imaginarios privados, tiene un impacto directo sobre la democracia ya que al no existir una imagen del nosotros, no permite la existencia de principios de solidaridad y voluntad colectiva para la integración de los ciudadanos.

La principal consecuencia de estos cambios es la desarticulación de los actores clásicos de la matriz estatal nacional popular y el surgimiento de una diversidad de actores, más distantes o también marginales al sistema político partidario, cuyas luchas se independizan entre sí, y en los cuales a los motivos políticos tradicionales, se suman nuevas temáticas que se derivan de cuestiones identitarias y de la búsqueda de autorrealización y felicidad

Este fenómeno debe insertarse en el contexto socio político más inmediato constituido por el proceso de democratización política que se inicia en $1990^{7}$. En diversos trabajos se ha llamado la atención sobre el hecho que este proceso en Chile fue exitoso en la medida que terminó con la dictadura, pero se trató de una democratización incompleta con enclaves autoritarios ${ }^{8}$, principalmente la institucionalidad legada por la dictadura y que tiene como elemento central la Constitución y sus leyes orgánicas constitucionales (entre las cuales está la referida a la educación Ley Orgánica Constitucional de Enseñanza -LOCE-) y otras herencias cuyo eje es el modelo socio-económico que los gobiernos democráticos de la Concertación 
de Partidos por la Democracia sólo corrigieron sin reemplazarlo. Una de las fórmulas políticas que usaron varios gobiernos de la Concertación, y de la cual el Consejo Asesor para la Calidad de la Educación que analizamos aquí es una expresión tardía, fue la llamada “democracia de los acuerdos”, que consistía en consensos entre gobierno y oposición de derecha tanto a nivel de directivas de partidos, comisiones o directamente en el Congreso. En realidad, no se trataba de acuerdos o consensos básicos sobre los fundamentos de un nuevo orden social democrático, sino de acuerdos tácticos o adaptativos que, en general, sólo corregían sin modificar sustantivamente los elementos heredados de la dictadura ${ }^{9}$.

\section{El sistema educacional y la herencia autoritaria}

Todo modelo educacional consolidado corresponde o está en consonancia con un modelo o proyecto de sociedad: En este sentido, el sistema educacional chileno fue generado bajo la dictadura militar para adecuarlo al modelo de sociedad, autoritario en lo político cultural y organizado en torno al individualismo y el mercado en lo económico social, que se buscaba implantar. A esta visión de sociedad, consagrada institucionalmente en la Constitución impuesta en 1980, correspondía una visión de la educación y una estructuración de ella que se anunciaron en las Directivas Educacionales de 1979 y en la legislación universitaria de 1981 y que cristalizaron, días antes del término de la dictadura, en la promulgación de la Ley Orgánica Constitucional de Enseñanza (Garretón, 2007).

Cuando se reinstala el régimen político democrático, los gobiernos de la Concertación no enfrentaron el modelo educacional como uno de los enclaves autoritarios de la dictadura que se debían eliminar (recién con Bachelet lo hacen y casi por obligación ante las movilizaciones estudiantiles), conectado con especialmente dos herencias fundamentales: la institucionalidad en el plano político y la desigualdad en el plano económico-social. A estas dos herencias, cabe agregar un tercer problema: las deficiencias para desarrollar un debate que aceptara los disensos de forma positiva de modo que pudieran ser procesados, no para transformarlos en consensos forzosos o superficiales, sino para integrarlos como diferencias positivas y facilitadoras de nuevos debates. Este tercer elemento dice relación con ese miedo al conflicto que se constata en el Informe PNUD (2002) y que incide en los miedos al debate, a declarar una posición disidente y que, como hemos dicho, se enfrentó a través de "la democracia de los acuerdos” que impedía la existencia de verdaderos debates sobre el fondo de las cuestiones. De hecho, muchos de quienes formarán parte del CAPCE, declaraban lo importante que era esta instancia por ser la primera vez que se hacía, señalando que sobre la educación el país "no debatía hace muchísimos años", "esta discusión no se hizo antes y aparece como necesaria” (El Mercurio, 27 agosto 2006).

Si bien la Concertación realizó grandes avances en la educación, estos fueron principalmente en el plano cuantitativo (cobertura, recursos, 
apoyo a establecimientos desfavorecidos), ya que en el plano cualitativo hay políticas cuyas consecuencias de implementación significaron aumentar la inequidad y segmentación del sistema (financiamiento compartido en el sistema subvencionado, por ejemplo). Y la institucionalidad del sistema permaneció la misma, estructurada, según el informe OECD de 2003, para generar desigualdades ${ }^{10}$.

\section{El movimiento estudiantil secundario del 2006, ¿nuevo movimiento social?}

No siendo nuestro objetivo la narración o el análisis del proceso denominado indistintamente "rebelión o revolución pingüina", baste con recordar que en Abril 2006 los dirigentes estudiantiles secundarios de los principales liceos del país, manifiestan sus peticiones en continuidad con planteamientos de años anteriores, especialmente el 2005 (OPECH, 2009; Garretón, 2007, Gonzalez, et al 2007). Sus principales demandas eran la gratuidad para la Prueba de Selección Universitaria (PSU) y el pase escolar, reformas a la Ley Orgánica Constitucional de Enseñanza (LOCE) y a la Jornada Escolar Completa (JEC), además de una serie de otras demandas vinculadas a la alimentación en los colegios, temas particulares de cada colegio en los planos de infraestructura, calidad del profesorado, etc. En el mes de Mayo, las marchas y tomas fueron en aumento, los primeros intentos de negociaciones con autoridades del Estado fracasaron. Luego, en nuevas negociaciones, lograrán que la agenda de corto plazo se cumpla: pase escolar con viajes ilimitados y becas de inscripción para la PSU a los alumnos de cuarto medio pertenecientes al $40 \%$ más pobre de la población. Sin embargo esto no depuso las movilizaciones, por lo que, ante el gran impacto mediático y el generalizado apoyo de la ciudadanía que suscitó el movimiento, la Presidenta Michelle Bachelet debió anunciar por cadena abierta de televisión el 1 de Junio de 2006, una serie de cambios que realizaría a la educación, más allá de las concesiones de la llamada agenda corta o de temas puntuales, incluyendo la conformación del Consejo Asesor Presidencial para la Calidad de la Educación, lo que abría una nueva vía de comunicación con el Estado, en que participarían representantes de diversos sectores sociales involucrados en la cuestión educacional ${ }^{11}$.

A nuestro juicio, estamos en presencia de un movimiento social, con densidad organizacional y temporalidad, mayores que un evento o un conjunto de movilizaciones. En efecto, con las estrategias de protestas, tomas, asambleas, jornadas de reflexión y la conformación del Bloque Social (con participación de representantes de padres y apoderados, docentes, co-docentes, estudiantes universitarios y técnicos, etc.) se marca una nueva forma de organización y participación, lo que también se refleja en los criterios que utilizaron para elegir los representantes y voceros del movimiento: territorialidad (norte-centro-sur del país y por organizaciones comunales), régimen educacional (público, priva- 
do y mixto) y también de género, dada la existencia de dirigentas mujeres con un papel muy activo en el movimiento.

Así, tal como en América Latina, en la que con posterioridad a las transiciones democráticas, el llamado a la sociedad civil es efecto de las insuficiencias de éstas y se expresa en nuevas formas de organización, no estamos en presencia de una lucha liderada por actores sociales tradicionales. Más bien, es un movimiento que convoca a otros sectores de la sociedad para que se hagan parte de sus demandas y expresen su malestar; a lo que se añade que canalizaron sus demandas por fuera del sistema político partidario. Ello se asocia a una nueva forma de politización, en donde los movimientos se vuelven más autónomos respecto de la política oficial: los estudiantes actuarían “frente” a ella y no “en” ella. (Garretón, 2007). Como vimos más arriba, tal fenómeno es propio de las formas subpolíticas de acción colectiva (Beck, 1998).

El hecho de que se organizaran en función de una "agenda corta" (por ejemplo pase gratuito y gratuidad de la PSU) y una "agenda larga" (por ejemplo, reforma a la LOCE y calidad de la educación) muestra asimismo que en sus acciones había una visión de largo plazo, por lo que también puede afirmarse que, junto al movimiento mapuche es el único movimiento social original de la época post dictatorial, comparable sólo al movimiento de derechos humanos que proviene de la época de la dictadura. Su particularidad como movimiento social, está el lograr vincular las tradicionales demandas micro o concretas, que coinciden con la "agenda corta”, con otras demandas valórico-institucionales, macro o estructurales que se vinculaban a la "agenda larga" de la educación. Así, como movimiento posee, a la vez una reivindicación específica en torno a la condición que se vive (como el pase escolar); una demanda de cambio institucional en un campo específico (la Ley Orgánica Constitucional de Enseñanza, LOCE); y una aspiración de cambio más global de la sociedad, usualmente de tipo utópico, que en este caso corresponde a que la reforma de la LOCE y las críticas a la municipalización y al lucro en la educación negocio implican la búsqueda de un nuevo sistema educacional y, por tanto, de un nuevo modelo económico social. (Garretón, 2007; González et al, 2007)

Los rasgos propios de un movimiento social explican por qué, pasado el momento de las movilizaciones, los estudiantes decidieron integrar y posteriormente continuar integrando el Consejo Asesor Presidencial, a pesar de las múltiples dudas que manifestaron hasta el final. Las movilizaciones estudiantiles no se depusieron en el periodo de funcionamiento del CAPCE, aunque sí disminuyó su presencia en prensa (El Mercurio, 26 de agosto 2006).Y si bien se retiran del CAPCE el día antes de la entrega del Informe Final, ellos se vieron siempre como contrapeso a las visiones más liberales sobre la educación, además de sentirse también gestores en la conformación de este consejo. Más adelante se profundiza en estas motivaciones. 


\section{El Consejo Asesor Presidencial para la Calidad de la Educación. ¿Nueva forma de hacer política?}

Como señalamos más arriba el movimiento de los secundarios correspondería, en clave de Holzer y Sørensen (2001), a una subpolítica activa, por conformar acción colectiva desde fuera del sistema político institucional y de forma políticamente intencionada. Y, en clave de Tilly y Tarrow (2007), a una política contenciosa transgresiva, por el carácter novedoso del actor estudiantil en el contexto de la sociedad postpinochetista. Y, precisamente, el CAPCE podría permitir la mantención del movimiento sin el desgaste propio de las movilizaciones. Porque una especificidad de la naturaleza de los movimientos estudiantiles es que no puede institucionalizarse y su horizonte temporal es muy corto, en la medida que los dirigentes estudiantiles terminan muy pronto su proceso escolar, en este caso en los meses siguientes. En la medida que el movimiento secundario obtuvo respuestas a sus propuestas más particulares, a través de la dinámica que les planteó el gobierno (reforma constitucional y CAPCE) sus metas de largo plazo podrían ser realizadas, aunque ahora dependería del gobierno y de otros poderes e intereses del campo educacional el que cumplieran.

Más allá de esta función política mencionada que cumpliría el CAPCE en relación al movimiento estudiantil, habría otros indicios para que éste y los otros Consejos Asesores Presidenciales creados durante el gobierno de Bachelet ${ }^{12}$, pudieran ser considerados como una nueva forma de hacer política. Porque si bien no se desliga plenamente del plano político, el Consejo se conformó como una entidad representativa de un "gobierno ciudadano" apelando al concepto acuñado durante la campaña presidencial $^{13}$ y es creado por la Presidenta, es decir, el Estado como una instancia de participación que nace frente a la política sin una institucionalidad previa. A ello se agregaría que entre los criterios para convocar a los consejeros, no se encontraba el de representatividad política tradicional: sólo dos de los más de 80 consejeros tenían cargos en el aparato central del Estado (una diputada y un senador) mientras que no más de cuatro tenían cargos en el aparato local municipal, ya fuesen alcaldes, encargados de corporaciones municipales, etc. Del lado de la sociedad civil, si bien los estudiantes no poseían los cupos que solicitaron originalmente (50\% más 1), si se suman todos aquellos cargos vinculados al llamado Bloque Social de la Educación, alcanzan un porcentaje similar. Este componente de la sociedad civil en el CAPCE, era el principal en términos numéricos y con personas de variadas tendencias políticas ${ }^{14}$. Si se considera que políticos y expertos, como ya indicamos (Beck, 1998) ya no tienen las respuestas a los cambios que vive la sociedad, sus incertidumbres deben compartirlas con ella, y esto es lo que ocurre en el CAPCE como lugar de encuentro entre políticos, sociedad civil y expertos.

Hay límites, sin embargo a esta nueva forma de hacer política si se le quiere considerar así. 
El primero radica en que, finalmente, la nominación de los integrantes es discrecional y no responde a la proporcionalidad de las posiciones expresadas por el movimiento estudiantil o por los diversos sectores que conforman el campo educacional.

El segundo es que si bien, de acuerdo a nuestras entrevistas, un aspecto muy valorado, en los inicios, es la búsqueda de solución del conflicto, tal solución, debido a las atribuciones y composición del Consejo, no puede ser una solución de fondo, sino que se busca o la desaparición o la recanalización del conflicto, en la medida que éste se percibe como una amenaza a la estabilidad del país, lo que lleva a que en los debates se busque evitar la manifestación de disensos. Se entendía que la finalidad del CAPCE era aunar visiones. Con el paso del tiempo de funcionamiento y el conocimiento entre los mismos consejeros, fueron agrupándose, emitiendo opiniones y disensos en conjunto. Pero la consecuencia no anticipada de todo ello es que las posiciones derrotadas por la movilización de los estudiantes (por ejemplo la crítica a la estructura municipal, la privatización y el lucro en la educación) y por la opinión pública tienden a relegitimarse y a imponerse al menos como veto a las transformaciones, con lo que esta dimensión de nueva forma de hacer política, pierde su sentido.

\section{El debate en el Consejo Asesor Presidencial}

Recordemos que el enclave educacional, institucionalizado y materializado en la Ley Orgánica (LOCE), constituía un respaldo cultural a la herencia socio-económica de la dictadura, es decir, era funcional al modelo denominado neo-liberal que ésta implantó. Lo que estaba en juego, entonces. como hemos mencionado, tanto en la movilización estudiantil como en las reacciones frente a ella y en la creación del CAPCE, era el cambio de la educación chilena, uno de los enclaves autoritarios más persistentes de la democracia inaugurada en 1990. A partir de ello, ¿̇en qué consistieron los temas tratados y los debates en el Consejo? ${ }^{15}$.

En cuanto a los temas tratados, hemos podido identificar siete grandes temas con sus respectivos subejes: finalidades de la educación (calidad, formación ciudadana, proyecto país); derechos y libertades (en la educación y la enseñanza, proyecto educativo); provisión educacional (pública/privada/subvencionada, solvencia municipal, municipalización); docentes (evaluación y estatuto docente, formación docente inicial y continua, carrera profesional y condiciones de trabajo; grupos vulnerables y territorio (etnias, necesidades educativas especiales, estudiante pobres y/o aislados); enseñanza superior y técnico-profesional; investigación sobre y en la educación. Es en torno a estos temas que se generan los acuerdos y disensos posteriores.

Si organizamos ahora los consensos y disensos, puede señalarse que con respecto a la Calidad de la Educación, hay gran consenso en el reconocimiento de los graves problemas que existen en el sistema educacional 
chileno y los consejeros concuerdan en la necesidad de precisar las metas o finalidades de una educación de calidad, las obligaciones del Estado y de las instituciones educacionales. Para generar un Sistema de Aseguramiento de la Calidad se propone reordenar las funciones del aparato estatal público a partir de las siguientes propuestas: responsabilidad del Ministerio de Educación en el diseño, financiamiento y ejecución de las políticas; restructuración y reforzamiento del Consejo Superior de Educación, que debe velar por la continuidad de las políticas educacionales, aprobar el currículum, los estándares para juzgar el desempeño del sistema y los requisitos de egreso de cada nivel de enseñanza; creación de una Agencia de Aseguramiento de la Calidad o Superintendencia de Educación que deberá evaluar el cumplimiento de los estándares de calidad a través de inspecciones, la administración de pruebas estandarizadas nacionales y comparativas internacionales, entregar a la ciudadanía información completa de los logros del sistema escolar y sugerir la salida del sistema o intervención de escuelas y liceos que no cumplan su rol.

Con respecto a la LOCE si bien no hubo unanimidad, sí hubo un acuerdo mayoritario en ésta que debía cambiarse ya que carecía de legitimidad social. De esta manera hubo acuerdo en que era necesario crear un nuevo marco regulatorio y una nueva ley que pusiera énfasis tanto en el derecho a la educación como en la calidad de ésta.

Con respecto a Financiamiento hay acuerdo entre los consejeros en que para recibir financiamiento público deben cumplirse con exigencias mayores que apuntan a los siguientes requisitos: naturaleza jurídica del establecimiento; garantías, representación y responsabilidad; requisitos académicos; aseguramiento de la calidad; obligación de informar y rendición de cuentas; reflexionar sobre la selección de alumnos y no permitir la discriminación arbitraria; contar con el personal acorde para el cumplimiento del proyecto educativo que se haya publicado; cumplir con las condiciones de equipamiento necesario que exige el proyecto educativo; no tener impedimentos para ser proveedor; autonomía.

En materia de docentes, hay acuerdo en el consejo de crear una carrera profesional docente y se proponen cambios curriculares para las instituciones que forman a los profesores. Se propone también la implementación de un examen de habilitación de carácter nacional y externo a las instituciones formadoras como requisito obligatorio para la obtención del título profesional, que se aplicaría al final de los estudios de formación.

En cuanto a los disensos, uno de los mayores disensos se dio en torno al rol de Estado. Identificando las posiciones, se encontraban, por un lado, quienes adherían a la mayor regulación por parte del Estado, siendo los más radicales en este sentido los estudiantes universitarios y secundarios, y, por otro, los más liberales como los representantes de los think tanks de derecha, de la educación particular o algunos expertos economistas. 
El lucro en la educación fue otro de los temas que provocó disensos al interior del Consejo. La mayoría de los consejeros rechaza la provisión por parte de las instituciones con fines de lucro, principalmente por razones éticas, pues la educación debe considerarse un bien colectivo incompatible con la mercantilización. Una posición rechaza la provisión educacional con fines de lucro cuando se recibe financiamiento público, ya que los fines de lucro podrían generar situaciones como que al tener la necesidad de maximizar utilidades la calidad de la educación se vea afectada. Un tercer grupo de consejeros señala que la provisión educacional con financiamiento público y fines de lucro permitiría ampliar la oferta educacional y con eso permitiría el libre ejercicio de la elección de educación a las familias, a lo que se agregaba que el sector privado actúa como proveedor en otros sectores vitales (salud, vivienda), a condición de que cumplan con ciertos requisitos legales y estándares de calidad definidos, por lo que el sector educación podría contar con los mismos mecanismos.

Con respecto al financiamiento hay disensos en las medidas que deben tomarse para mejorar el sistema. Las propuestas se orientan en dos líneas diferentes. La primera, denominada "Fortalecimiento del sistema de subvenciones", plantea un conjunto de medidas para mejorar el actual sistema, tales como: aumentar el monto de la subvención según criterios como los distintos niveles, modalidades de enseñanza, zona geográfica, necesidades educacionales especiales, inclusión de niños de pueblos indígenas, etc; subvención diferenciada en función de las características socioeconómicas; subvención por mantenimiento de la infraestructura, equipamiento, más la subvención por escolaridad; consideración de matrícula en el pago de subvención; máxima autonomía en el uso de los recursos de la subvención; financiamiento de regulaciones externas a los sostenedores; financiamiento de los administradores de la educación estatal. La otra alternativa, proviene del sector más crítico al actual sistema de financiamiento. Esta propuesta, denominada "Esquema de financiamiento mixto", básicamente propone: un financiamiento fijo para las entidades administradoras de educación estatal, según las características de la población y sus escuelas; monto fijo para las escuelas del sistema estatal y los establecimientos de propiedad privada que cumplan con la condición de igualdad de trato (no seleccionar y no expulsiones arbitrarias); una fracción con financiamiento de subsidio a la demanda, especialmente a aquellos establecimientos que atienden a estudiantes con mayores dificultades en el proceso educativo.

Una de las principales polaridades del debate se produjo en torno al derecho a la educación vs la libertad de enseñanza. Este eje atravesó la discusión en torno a la LOCE así como respecto del proyecto educativo a nivel del establecimiento educacional, lo que implicaba la reflexión en torno a dos enclaves, el institucional, por cuestionar la LOCE , y el cultural por cuanto los proyectos educativos definen los lineamientos de los establecimientos sobre las finalidades y modalidades de la educación.

Según la prensa, a las dos semanas de conformado el CAPCE existían los primeros acuerdos logrados en esta materia, lo que mostraba la 
posibilidad de que los polos ideológicos que integraban el CAPCE dialogaran y sentaran bases comunes: se había acordado la necesidad de garantizar jurídicamente el derecho a una educación de calidad y ello no era incompatible o no afectaba la libertad de enseñanza. El Coordinador de la Comisión de Marco Regulatorio diría al respecto: “Que se haya alcanzado esa convergencia en una comisión tan heterogénea es bien importante. La libertad de enseñanza y el derecho a la educación no están en colisión. O sea, no es cierto que todo lo que se fortalezca la libertad de enseñanza vaya en desmedro del derecho a la educación o que fortalecer el derecho a la educación estropea la libertad de enseñanza” (El Mercurio, 02 de julio 2006). En otra ocasión, el consejero y Presidente de la Federación de Instituciones de Educación Particular (FIDE), Jesús Triguero, en palabras de El Mercurio declararía que "la libertad de enseñanza y el derecho a la educación 'pueden y deben coexistir' [...] para cumplir este derecho se debe ofrecer una multiplicidad de proyectos educativos, de modo que los padres puedan elegir la educación que los jóvenes necesitan. Este derecho de los padres no es posible de cumplir si no existe libertad de enseñanza” (El Mercurio, 19 de julio 2006).

También puede señalarse la existencia una mirada técnica que genera más acuerdos y una mirada político-ideológica que provoca disensos y, por otro lado, una tensión entre lo político-social versus una mirada económica, lo que se vincula con la dicotomía anterior, pues muchas veces la mirada técnica va acompañada por una economización de los temas en oposición a una sociologización de ellos. De hecho, si se examina la lista de los consejeros "expertos” la mayoría proviene del mundo de la economía y escasamente desde de la sociología u otras ciencias (medicina, ingenierías).

En términos políticos ideológicos, los disensos se organizaron en torno a los derechos versus las oportunidades. Así, por un lado, una mirada enfatiza el garantizar una educación de calidad para todos y, por otro lado, otra enfatiza la igualdad de oportunidades en el acceso a la educación, lo que refiere a la clásica distinción entre el Estado de Derechos, de corte más social demócrata vs la sociedad de oportunidades de sesgo más liberal.

La dicotomía clásica Estado-Mercado también se reproduce al interior del CAPCE. Podemos hablar de miradas estatistas y miradas liberales, donde las primeras pretendían la vuelta al llamado Estado Docente y las segundas buscaban que se mantuviera el sistema educativo actual, teniendo el Estado como única responsabilidad compensar las insuficiencias del mercado. Opiniones de consejeros reflejan este eje. Por ejemplo, la de un representante de colegios particulares: "Ese también fue otro tema que generó como harta discusión, sobre como tenía que ser, porque en realidad lo que estábamos discutiendo ahí nuevamente era la discusión entre mercado y Estado, porque un esquema en donde... la demanda del alumno, es un esquema mucho más de mercado, en que los papás eligen, y los papás dan a elegir, generan competencia, y la gente, el sector decía yo no quiero competencia, pero cómo validamos eso con que los papás elijan, porque a la 
gente igual le gusta el tema de elegir, el hecho de tener la opción de elegir da mayor bienestar que no hacerlo... y en nada tampoco soluciona el problema de la segregación, entonces se empezaban a armar todas las líneas de argumentación, quizás ese fue un tema que generaba como mayor disenso" (Representante de colegios particulares). O la de un experto "Es que hay tantos matices verdad. Cuando digo estatista, yo percibía que habían unas personas que eran muy nostálgicas de una situación en cuanto a presencia, regulación del Estado, etcétera [...] Yo estudiaba en un colegio privado que era excelente académicamente, pero tenía comisiones examinadoras externas que venían de colegios públicos, o en la universidad, yo me recibí de médico el año 66, y mi examen de grado y mi título de grado fue otorgado por la Universidad de Chile. Yo creo que esas cosas eran anacrónicas, pero habían personas que de alguna manera manifestaban nostalgia por ese rol del Estado. Y había otras personas que consideraban que cualquier atribución adicional a las que tiene ahora el Estado era una amenaza para la educación privada. Entonces, yo diría que esos eran los dos polos, y en algunas cosas yo estaba de acuerdo con un grupo, en otras con el otro. Pero si yo tuviera que definir digamos, dos grandes facciones digamos, encontradas en muchos puntos, eran esas”

Más allá de estas polaridades, ¿pudo generarse un consenso fundamental en torno a las transformaciones del modelo educacional chileno? A partir de la investigación sobre los Consejos de la que forma parte este artículo $^{16}$, puede afirmarse que los grandes acuerdos o consensos a los que han llegado los Consejos Asesores conformados durante el mandato de la presidenta Michelle Bachelet, se mantienen en un grado de superficialidad correspondiendo a consensos en los diagnósticos, no reconociéndose cuáles son las soluciones a los problemas más graves o profundos que afectan al país. Así, en el CAPCE, los consejeros coincidieron en la necesidad y urgencia de mejorar la calidad de la educación. Pero como señala una entrevistada, que corresponde a la categoría de miembro experta del Consejo, al responder sobre cuáles son los temas en que se llegó a mayor consenso en particular: "Yo creo que siempre es el diagnóstico, las debilidades que tiene nuestro sistema educativo con financiamiento público, hay un consenso diría transversal, que el financiamiento es escaso y que con treinta mil pesos no se educa a nadie y también en entregar más, pero también entregar más recursos a cambio de mejoras y ahí como que la derecha siempre ha tenido la postura de que la Concertación ha aumentado, ha duplicado el presupuesto en educación y no se han visto resultados en términos de calidad y yo creo que nosotros hacemos un poco el mea culpa de que eso es así que hay que generar el quiebre. En el cómo generar ese quiebre ahí empiezan todas las divergencias”. Esta misma visión queda plasmada en los voceros de la Asamblea Coordinadora de Estudiantes Secundarios (ACES), quienes luego del primer Informe de Avance del Consejo, declaran que "El Consejo sólo ha servido para analizar cuál es la problemática de la educación, que ya todos teníamos clara” (El Mercurio, 01 octubre 2006)

En este sentido, independientemente del sector político con el que se simpatizara o militara, los consejeros coincidieron en que había que cam- 
biar la situación. Algunos consejeros señalaron en las entrevistas que la LOCE era una herencia de la dictadura (enclave institucional en nuestras palabras), especialmente estudiantes, profesores y algunos expertos; para otros, sólo se debía cambiar algunos aspectos de esta ley, no siendo necesaria su derogación como pedían los estudiantes. De modo que hubo consenso en el CAPCE respecto de cambiar la LOCE, ya fuera mediante modificaciones o derogación, lo que respondía nuevamente a la pregunta del qué es lo que está mal, pero las posturas divergentes vendrán en las preguntas posteriores del por qué la educación llegó a esta situación y en cómo solucionarlo.

En cuanto a la pregunta del por qué la educación estaba en crisis, ella genera una diferencia significativa, pues mientras algunos señalaban que las falencias actuales de la educación se debían a una herencia de la dictadura, otros consejeros mencionaban que la causa eran las políticas educacionales de la Concertación. Por ejemplo, un consejero dirigente de profesores mencionó en las entrevistas "Se confrontaban dos visiones, entre aquellos que señalaban que los problemas que tenía la educación chilena eran producto de que no se había logrado despejar la estructura que había dejado Pinochet instalada, y por lo tanto, a partir de eso era lo que provocaba los problemas, y eso era lo que había que hacer, y aquellos otros que indicaban que la reforma educacional de los gobiernos de la Concertación no había sido lo suficientemente eficaz, y por lo tanto que había que reformar lo que se había hecho dentro del mismo gobierno”.

Las dificultades de llegar a consenso de fondo tienen que ver también con la representatividad de los consejeros. Teóricamente, los consejeros convocados, no "representaban" a ningún sector, es decir, ninguno de ellos era portavoz oficial de un grupo, aunque sí expresaran puntos de vista asimilables a posiciones sectoriales. Esta individualidad refleja la dificultad de lograr un acuerdo cuya estabilidad en el tiempo permitiese cambios estructurales.

A diferencia de ello, como hemos indicado más arriba, los estudiantes tenían esa visión de conjunto para cambiar y mejorar la educación, por lo que hemos afirmado su capacidad como movimiento social, lo que los llevó, terminadas las movilizaciones, a participar en el CAPCE y, presionar, una vez obtenidas las metas de la agenda más puntual, por cambios estructurales y "evitar que posturas conservadoras presionen y obtengan mayor representación que nosotros", porque "ahora nuestra estrategia apunta a generar presión a nivel político para lograr que el próximo año el Congreso apruebe los cambios estructurales" (El Mercurio, 09 de octubre 2006). Entre las razones que expusieron los estudiantes para mantener en el CAPCE, se encuentra, primero, que la creación del Consejo de Educación fue un logro de los estudiantes, y era un sin sentido retirarse. Segundo, que era una forma de presionar en las políticas públicas, en la medida que no tenían representación en el Congreso de la República. Tercero, amortiguar al bloque conservador presente en el CAPCE. 
La imposibilidad de generar cambios estructurales mediante estas “mesas” (Correa, 2008), se refleja en el tema de la educación superior. El fue discutido hacia el final, en una sub-comisión, pero una serie de críticas y declaraciones dejaron sin efecto sus propuestas, lo que llevó a la creación de un nuevo Consejo especializado (El Mercurio, 07, 09 y 12 de diciembre 2006)

El resultado fue que el Informe Final del Consejo incluyó todas las posiciones planteadas, pero sin definición ni pronunciamiento sobre la reforma a la estructura y gestión del sistema escolar público, el efectivo papel rector del Estado o el lucro en la educación privada financiada por el Estado, por lo que la definición de políticas y reformas legales quedaba entregada a negociaciones posteriores ${ }^{17}$. Así, con motivo de la entrega del Informe en Diciembre de 2006, la Presidenta se refirió a tres ejes de reforma. El primero era la sustitución de la LOCE y el proyecto de la Superintendecia de Educación. El segundo eje correspondía al sistema de financiamiento, el cual se cambiaría para generar equidad entre los alumnos con la subvención preferencial, además de aumentar la rigurosidad y transparencia en la entrega de recursos a través de exigentes rendiciones de cuentas. El último eje al cual aludió, fue el fortalecimiento de la educación estatal por medio de "un adecuado sistema de gestión escolar e institucionalidad”. En Noviembre de 2007 se firmó el "Acuerdo por la calidad de la educación” entre el gobierno, la oposición de derecha y la Concertación, con el rechazo de los actores estudiantiles y docentes, lo que cristalizó en la promulgación de la Ley General de Educación en Septiembre de 2009 que reemplazaba la LOCE, pero que dejaba sin resolver el problema de la educación pública, que quedó entregado a una iniciativa legal que nunca prosperó, y la estructura y gestión del sistema educacional.

\section{Consideraciones finales}

No ha sido el objeto de este artículo un análisis del sistema educacional chileno, sino de mostrar cómo en una situación post transicional surgen movimientos sociales y formas de subpolítica que buscan su transformación y cómo las soluciones que buscan consensos, como un Consejo Asesor, logran resolver los conflictos desatados por las movilizaciones pero no así los problemas de fondo que los suscitaron.

No puede desconocerse que el sistema educacional chileno cambió a raíz de las movilizaciones estudiantiles del año 2006. Pero tampoco puede afirmarse que el sello fundamental del modelo heredado de la dictadura fue superado.

En este sentido, puede sostenerse que las propuestas del CAPCE fueron derivadas más como estrategias políticas y no como reformas institucionales, correspondiendo a una nueva versión de los que se llamó la democracia de los acuerdos y no a consensos básicos de cambios más profundos. El carácter meramente consultivo de esta instancia refuerza este 
aspecto $^{18}$. Ello parece confirmar que la alusión al “gobierno ciudadano” de la Presidenta Bachelet no logró transformarse en un sistema institucional para el diálogo entre la ciudadanía y la política.

Estos Consejos Asesores, en especial el CAPCE, fueron instancias ad hoc y de duración acotada, y no generaron un hilo conductor de las políticas públicas de modo que ellas tuvieran continuidad y se enmarcaran en proyectos de largo plazo. El gobierno de Bachelet era un gobierno que generaba el espacio de diálogo, lo promovía, pero sin un proyecto de sociedad que incluyera a la educación en su agenda y menos a los estudiantes movilizados.

En definitiva, en el sustrato de las decisiones y resultados de esta nueva forma de hacer política primó la intangibilidad de un modelo societal en el que se basa el modelo educacional. Esta visión de sociedad, que no pone en el centro el carácter de enclave autoritario del modelo educacional, fue la que predominó y está en el origen de la falta de acuerdos estructurales y de un hilo conductor de transformaciones profundas. 


\section{Notas}

${ }^{1}$ Este artículo se enmarca en el desarrollo del proyecto Fondecyt № 1090127 titulado "Movilizaciones Sociales, Estado de Bienestar y Conocimiento Experto. El Significado de los Consejos Asesores Presidenciales", cuyo investigador responsable fue Manuel Antonio Garretón y los coinvestigadores María Angélica Cruz Contreras y Félix Aguirre Díaz. Participaron en el proyecto en calidad de Asistentes Javiera Araya, Naim Bro, Francisco Espinoza, Elias Farias, Pierina Ferreti, David Mally, Tamara Ramos. Junto al análisis documental, se realizaron entrevistas individuales y grupales a actores participantes de los Consejos o involucrados indirectamente por sus debates y decisiones. En este artículo nos hemos circunscrito al Consejo de Calidad de la Educación.

${ }^{2}$ Por los colores del uniforme de los estudiantes

3 Ver un detalle de estas conceptualizaciones en los textos de M.A.Garretón (1996, 2001,2007), el primero de índole general y los otros dos para América Latina y Chile respectivamente. Mucho de estas conceptualizaciones, se basan libremente en la perspectiva de Alain Touraine sobre movimientos sociales. Entre las más recientes Touraine (1997).

${ }^{4}$ Entre las múltiples visiones sobre el concepto de sociedad civil, una para el caso latinoamericano A. Panfichi (2002) y una más reciente sobre diversos contextos socio-históricos, B.Jobert y B.Kohle. eds (2008).

${ }^{5}$ Véase sobre la discusión conceptual de políticas públicas Jobert (2004), Oszlak (2007). Para el caso chileno, De la Maza (2005, 2009)

${ }^{6}$ Basamos lo que sigue especialmente en Garretón, (1995, 2000) y Garretón et. al. ( 2004).

${ }^{7}$ Recordemos que la dictadura militar encabezada por Pinochet con apoyo de la derecha chilena rigió desde 1973 a 1990, siendo sucedida por gobiernos democráticos electos de la coalición de centro-izquierda, la Concertación, de Partidos por la Democracia, (en adelante la Concertación), de Patricio Aylwin (1990-1994), Eduardo Frei Ruiz Tagle (1994-2000), Ricardo Lagos (2000-2006) y Michelle Bachelet (2006-2010). En 2011 asumió el gobierno de derecha presidido por Sebastián Piñera.

${ }^{8}$ El concepto de enclave autoritario alude a elementos institucionales, ético-simbólicos, actorales y culturales que son propios de un régimen autoritario pero que quedan incrustados en el régimen democrático dándole el carácter de democracia incompleta. Ver Garretón M.A. y Garretón, R 2010

${ }^{9}$ Una visión crítica de esta política en Garretón 2000

${ }^{10}$ Sobre el sistema educacional chileno y las políticas de los gobiernos de la Concertación ver, entre otros, OECD (2003).

${ }^{11}$ Una cronología y análisis del movimiento estudiantil, en OPECH (2009)

${ }^{12}$ Sobre los Consejos Asesores creados por Bachelet, ver Aguilera (2009)

${ }^{13}$ Ver una crítica a este respecto en Garretón (2010) 
${ }^{14}$ Si se considera que la primera nómina del CAPCE poseía 74 convocados, los 12 cupos concedidos estaban lejanos a la cifra que querían los estudiantes (un $17 \%$ del 50\% más uno). Los 12 cupos para los estudiantes, se dividieron de tal manera: 6 cupos para secundarios (3 para la Región Metropolitana y 3 para regiones) y otros 6 para universitarios (3 de entidades públicas y 3 para privadas). Cabe destacar que también primó -de forma consciente o inconsciente- el criterio de representación por zona de regiones, pues se incluyeron universidades y estudiantes del norte, centro y sur del país (U. Católica del Norte, U. Federico Santa María de Valparaíso y U. de Bío Bío, mientras que los estudiantes venían de Valparaíso, Concepción) ( Diario El Mercurio, 07 de junio y 11 de junio 2006). El detalle de la composición del Consejo en Consejo Asesor Presidencial para la Calidad de la Educación (2006).

${ }^{15} \mathrm{El} \mathrm{CAPCE}$ se estructuró en tres subcomisiones que se organizaron en grupos más pequeños que discutían a partir de preguntas para sus ámbitosespecíficos. Ellas fueron: Marco Regulatorio, Institucionalidad y Calidad. Posteriormente, se crearon una subcomisión de Educadores/Docentes y otra de Educación Superior, pero al tener integrantes de otras subcomisiones, su forma de funcionamiento difería a las primeras subcomisiones formadas. Ver Consejo Asesor... (2006) op.cit. En lo que sigue presentamos la ordenación de temáticas, consensos y disensos de acuerdo a nuestra propia investigación

\footnotetext{
${ }^{16}$ Ver nota inicial

${ }^{17}$ Ver el Informe Final en Consejo Asesor Presidencial (2006) op.cit.
}

18 Así, los entrevistados aceptan que su deber como consejeros era precisamente aconsejar, siendo las instancias parlamentarias y estructuras gubernamentales (ministros, presidentes) los que deben decidir, pese a lo cual, sentían que su trabajo habría sido más provechoso si el carácter de los consejos fuese, al menos, vinculante. 


\section{Bibliografía}

Aguilera, C. (2009), Un análisis de las comisiones asesoras presidenciales del gobierno de Michelle Bachelet, FLACSO, Santiago de Chile.

Beck, U. (1998), La invención de lo político, FCE, Buenos Aires.

Castel, R. (2004), La Inseguridad Social, Manantial, Buenos Aires.

Consejo Asesor Presidencial para la Calidad de la Educación (2006), http:/ /www.consejoeducacion.cl

Correa, S. (2008), "El corporativismo como expresión política del socialcristianismo”, en Teología y Vida vol $48 n^{\circ} 3$, pp 467 - 481, Pontificia Universidad Católica de Chile, Santiago de Chile.

De la Maza, G (2005), Tan lejos, tan cerca. Políticas públicas y sociedad civil en Chile, LOM Ediciones, Santiago de Chile.

Ídem (2009), "La institucionalización de la participación en las políticas públicas en Chile. Estado actual y desafíos”, en Fuente, G. et.al. Participacion y nuevos desafios politico-institucionales, Ministerio Secretaría General de la Presidencia, Santiago de Chile.

Garretón, M. A. (1983), El Proceso Político Chileno, FLACSO Santiago de Chile.

Ídem (1996), "Social Movements and the process of democratization. A general framework", en International Review of Sociology, Vol 6, n ${ }^{\circ} 1$, Universidad de Roma, Roma.

Ídem (1995), Hacia una nueva era política. Estudio sobre las democratizaciones, FCE, Santiago de Chile.

Ídem (2001), Cambios sociales, actores y acciones colectivas en América Latina, Serie Políticas Sociales, CEPAL, División de Desarrollo Social, Santiago de Chile.

Ídem (2000), La sociedad en que viviremos. Introducción sociológica el cambio de siglo, LOM Ediciones, Santiago de Chile.

Ídem (2007), Del postpinochetismo a la sociedad democrática. Globalización y política en el Bicentenario, Editorial Debates, Santiago de Chile.

Ídem (2010), "La leadership presidenziale di Michele Bachelet: stile, progetto, popolarità" en D. Campus (ed.) L'immagine della donna leader, Bononia University Press, Bologna. Disponible en www.manuelantoniogarreton.cl. 
Garretón, M.A, Cavarozzi, M., Cleaves, P., Hartlyn, J., Gereffi, G. (2001), América Latina en el siglo XXI, Hacia una nueva matriz socio-política, LOM Ediciones, Santiago.

Garretón M.A. y Garretón R. (2010), "La democracia incompleta en Chile: La realidad tras los rankings internacionales”, en Revista de Ciencia Política Vol.30, No 1, Pontificia Universidad Católica de Chile, Santiago de Chile.

Giddens, A. (1998), The Third Way. The Renewal of Social Democracy. Cambridge Polity Press, Cambridge.

González, J., Cornejo, R., Sánchez, R. y Caldichoury, J. P. (2007), Perspectivas y significados del movimiento nacional de estudiantes secundarios chilenos, OPECH, Universidad de Chile, Santiago de Chile.

Holzer, B. y Sørensen, M. (2001), Subpolitics and Subpoliticians, Arbeitspapier 4 des SFB 536 Reflexive Modernisierung, München.

Jobert, B. (2004), Estado, Sociedad, Políticas Públicas. LOM Ediciones, Santiago de Chile.

Jobert, B. y Kohle, B. (eds) (2008), Changing images of civil society: From protest to governance, Routledge, Nueva York.

Lechner, N. (1994), “Los nuevos perfiles de la política. Un bosquejo” en Nueva Sociedad $n^{\circ} 130$, Fundación Friederich Ebert, México D.F.

Mc Adam, D., Tarrow, S. y Tilly, Ch. (2004), Dynamics of Contention, Cambridge University Press, Cambridge.

OECD (2003), Informe sobre la Educación Chilena, OCDE, Santiago de Chile.

OPECH (2009), De actores secundarios a estudiantes protagonistas. Disponible en: www.opech.cl

Oszlak, O. (2007), “Formación histórica del Estado en América Latina: elementos teórico-metodológicos para su estudio”, en C. Acuña (comp.) Lecturas sobre el Estado y las políticas públicas: Retomando el debate de ayer para fortalecer el actual, CEDES, Buenos Aires.

Panfichi, A. (2002), Sociedad civil, esfera pública y democratización en América Latina: Andes y Cono Sur, FCE, México D. F.

PNUD (2002), Informe de Desarrollo Humano. Nosotros los chilenos, un desafío cultural, PNUD, Santiago de Chile. 
Polis, Revista de la Universidad Bolivariana, Volumen 10, $N^{\circ}$ 30, 2011

Tilly, Ch. y Tarrow, S. (2007), Contentious politics, Paradigm Publishers, Londres.

Touraine, A. (1997), ¿Podemos vivir juntos? Iguales y diferentes, FCE, México D.F.

Recibido: 10.06.2011

Aceptado: 23.11.2011 